Diagnostic Methods

\title{
Concurrent validity and within-session reliability of gait kinematics measured using an inertial motion capture system with repeated calibration
}

\author{
Karina Berner ${ }^{\mathrm{a},{ }^{*},}$, John Cockcroft ${ }^{\mathrm{b}}$, Linzette D. Morris ${ }^{\mathrm{c}}$, Quinette Louw ${ }^{\mathrm{a}}$ \\ a Stellenbosch University, Faculty of Medicine and Health Sciences, Division of Physiotherapy, PO Box 241, Cape Town, 8000, South Africa \\ b Stellenbosch University, Central Analytical Facilities, Neuromechanics Unit, Private Bag X1, Matieland, 7602, South Africa \\ ${ }^{\mathrm{c}}$ Department of Physical Therapy \& Rehabilitation Science, College of Health Sciences, QU Health, Qatar University, Doha, Qatar
}

\section{A R T I C L E I N F O}

\section{Article history:}

Received 18 June 2019

Received in revised form

19 May 2020

Accepted 16 June 2020

\section{Keywords:}

Accuracy

Repeatability

Inertial measurement unit

Gait kinematics

Single-pose calibration

Reference pose

Biomechanics

\begin{abstract}
A B S T R A C T
Introduction: Wearable inertial measurement units (IMUs) enable gait analysis in the clinic, but require calibrations that may affect subsequent gait measurements. This study assessed concurrent validity and within-session reliability of gait kinematics measured by a frequently calibrated IMU-based system. Calibration pose accuracy and intra-rater repeatability, and IMU orientation tracking accuracy, were additionally quantified.

Methods: Calibration poses and gait were recorded in 15 women using IMUs and optical motion capture (OMC) (reference standard) simultaneously. Participants performed six consecutive trials: each comprising a calibration pose and a walk. IMU tracking was assessed separately (once-off) using technical static and dynamic tests. Differences of $>5^{\circ}$ constituted clinical significance.

Results: Concurrent validity for gait revealed clinically significant between-system differences for sagittal angles (root-mean-square error [RMSE] $6.7^{\circ}-15.0^{\circ}$; bias $-9.3^{\circ}-3.0^{\circ}$ ) and hip rotation (RMSE $7.9^{\circ}$; bias $-4.2^{\circ}$ ). After removing modelling offsets, differences for all angles (except hip rotation) were $<5^{\circ}$. Gait curves correlated highly between systems ( $r>0.8$ ), except hip rotation, pelvic tilt and -obliquity. Within-session reliability of IMU-measured gait angles was clinically acceptable (standard error of measurement $[\mathrm{SEM}]<5^{\circ}$ ). Calibration poses were repeatable (SEM $0.3^{\circ}-2.2^{\circ}$ ). Pose accuracy revealed mean absolute differences (MAD) $<5^{\circ}$ for all angles except sagittal ankle, hip and pelvis. IMU tracking accuracy demonstrated RMSE $\leq 2.0^{\circ}$.

Conclusion: A frequently calibrated IMU system provides reliable gait measurements; comparing highly to OMC after removing modelling differences. Calibration poses can be implemented accurately for most angles and consistently. IMU-measured gait data are clinically useful and comparable within participants, but should not be compared to OMC-measured data.
\end{abstract}

() 2020 Elsevier Ltd. All rights reserved.

\section{Introduction}

Gait assessment is an inherent part of evaluating movement disorders. Clinical evaluation has traditionally involved semiquantitative human observations. However, such assessments may be oversimplified. Early, subtle locomotor deteriorations can occur without obvious pathology (de Bruin et al., 2008) and may be overlooked by the human eye (Chen et al., 2016). Quantitative gait

\footnotetext{
* Corresponding author.

E-mail addresses: kberner@sun.ac.za (K. Berner), johnc@sun.ac.za (J. Cockcroft) Imorris@qu.edu.qa (L.D. Morris), qalouw@sun.ac.za (Q. Louw).
}

analysis using instrumented motion capture can provide better accuracy and precision (Cuesta-Vargas et al., 2010) as 3D movements are tracked and captured in high resolution. Motion capture is particularly useful for systematically quantifying the overall state (quality) as well as specific parameters of walking gait, and can distinguish pathologic patterns (Cimolin and Galli, 2014; CuestaVargas et al., 2010). Using quantitative data may enable better decision-making by supporting clinical reasoning (Chen et al., 2016). Indeed, quantitative gait parameters have been emphasized as functional markers in various populations such as gross neurological (Roiz et al., 2010), musculoskeletal (Astephen et al., 2008), and endocrine (Fernando et al., 2015) conditions, sports- 
related injuries (Leibbrandt and Louw, 2017) and the elderly (Begg and Sparrow, 2006); and fall prediction models based on common clinical risk assessments (questionnaires and physical tests) have been shown to improve when measures from a comprehensive quantitative gait analysis are included (Doi et al., 2013; van Schooten et al., 2015).

The current reference standard in motion capture technology is optical motion capture (OMC) (Ancillao, 2016). However, OMC's clinical appeal is hampered by issues including high costs, expert operation requirements, cumbersome setup and post-processing procedures, marker occlusion and limited ecological validity due to being laboratory-restricted (Ali et al., 2012; Iosa et al., 2016). Thus, despite providing high quality data, OMC presents an access barrier to clinicians and patients. More pragmatic alternatives are therefore increasingly being investigated.

As technology supporting motion capture has advanced dramatically over the past couple of decades, in-field quantitative gait analysis using portable motion capture systems has become a possibility. Inertial motion capture systems using inertial measurement units (IMUs) are increasingly used in research and clinical practice (Picerno, 2017). Relative to OMC, IMU-based systems are compact, portable, affordable and user-friendly; increasing clinical appeal and ecological validity (Iosa et al., 2016). Using sensor fusion techniques, the IMU sensors - comprising on-board accelerometers, gyroscopes and magnetometers - can track orientation accurately (Balasubramanian, 2013). However, IMU-based systems suffer from various sources of error. For example, IMUs experience time-increasing drift in the presence of ferromagnetic disturbances, necessitating controlled test protocols. Another major technical limitation is the inability of IMUs to directly measure absolute skeletal position kinematics. Therefore, unlike with OMC systems, information about skeletal posture is not directly available to IMU-based systems.

One workaround solution is to determine IMU orientations relative to body segments during a calibration procedure. Only after model calibration, can estimates of joint/segment kinematics (linear translation) be made via mathematical techniques (Iosa et al., 2016). Various calibration approaches exist, ranging from simple static single- or double-poses, to more complex dynamic functional calibrations (Picerno, 2017). Single-pose calibrations, involving determination of IMU orientations relative to a reference posture (Picerno, 2017), are often utilized by commercial inertial motion capture systems as they are quick and easy to implement. The accuracy and repeatability of calibration poses may be compromised by skeletal alignment constraints, implementation technique (e.g. self- versus instructed placement) and instructor skills in setting up the posture; and errors introduced during calibration may affect subsequent kinematic measurements. RobertLachaine et al. (2017) demonstrated that passive placement of a static calibration pose results in better accuracy than selfplacement and improves similarity between IMU and OMC models. They reported that such poses were repeatable (standard error of measurement [SEM] ranging from $1.5^{\circ}$ to $2.1^{\circ}$ ); however, they did not measure subsequent gait kinematics.

Importantly, differences in joint/segment definitions mean that OMC biomechanical models typically demonstrate angular offsets from the assumed IMU reference neutral pose (Nüesch et al., 2017; Robert-Lachaine et al., 2017). Although IMU-measured range of motion (ROM) and movement patterns do not seem to be significantly affected, absolute values may not be directly comparable between systems/models (Nüesch et al., 2017; Picerno, 2017; Seidel et al., 2015). Previous studies have reported promising but varying results regarding the concurrent validity of IMU- versus OMCmeasured gait kinematics. Some authors have demonstrated improved comparability between IMU and OMC systems/models after removing biomechanical model offsets (Cloete and Scheffer, 2008; Nüesch et al., 2017). Without removing modeling differences, Seidel et al. (2015) found that OMC produced higher absolute kinematic gait angles relative to an IMU system (differences ranging from $-4.2^{\circ}$ to $10.8^{\circ}$ ) although the systems were highly correlated (0.73-0.98).

Confirming the validity and reliability of IMU-based systems to investigate human locomotion is imperative to future applications outside of the laboratory. Real effects identified by IMUs should be separable from system measurement errors. For instrumented motion capture, absolute errors of $<5^{\circ}$ have been proposed as clinically reasonable (McGinley et al., 2009). The primary aim of this study was to determine the concurrent validity of clinical gait analysis outcomes measured by a commercial IMU system, using OMC and the Convention Gait Model (CGM) as reference. It was hypothesized that IMU-measured gait kinematics would correlate highly to OMC measurements, but would demonstrate low concurrent validity due to differences between the assumed and actual reference calibration postures. Secondly, this study aimed to determine the within-session reliability of IMU-measured kinematic angles, using six repeated gait trials. The secondary objectives were to use OMC to quantify the accuracy and consistency of two factors (other than modelling) affecting inertial motion capture validity and reliability: reference-pose implementation by a trained instructor, and IMU sensor orientation tracking.

\section{Methods}

\subsection{Participants}

The study was conducted according to the Declaration of Helsinki. The Stellenbosch University Health Research Ethics Committee granted ethical approval (N15/05/043). A convenience sample of 15 healthy able-bodied adult female volunteers (age: $20.93 \pm 2.46$ years; height: $1.63 \pm 0.08 \mathrm{~m}$; BMI: $21.74 \pm 1.59 \mathrm{~kg} / \mathrm{m}^{2}$ ) signed written informed consent. Participants with a BMI $>25 \mathrm{~kg} /$ $\mathrm{m}^{2}$ were excluded.

Sample size was based on the SEM for lower limb kinematic angles across the gait cycle, considering an SEM of $4.1^{\circ}$ (Meldrum et al., 2014). This was the maximum SEM (hip rotation) reported across tri-planar lower limb angular ROM in a healthy population during habitual walking. To establish that a measured SEM of $4.1^{\circ}$ is lower than a minimum clinically important difference (MCID) of $5^{\circ}$ (McGinley et al., 2009) at a one-sided 95\% confidence interval (CI), the recommendations by Stratford and Goldsmith (1997) were followed: the variance ratio $\left(\frac{\sigma^{2}}{s^{2}}\right)$ was calculated as $\left(\frac{5.0^{2}}{4.1^{2}}\right)=1.5$, and the required sample size was estimated for a protocol using 4 to 6 measurements per participant. A sample size of 9 to 15 was thus deemed appropriate.

\subsection{Instrumentation}

Three-dimensional trajectories were captured simultaneously using a seven-IMU inertial motion capture system (myoMOTION Research Pro, Noraxon USA Inc.) and an eight-camera OMC system (MX T-series, VICON Motion Systems Limited). A sampling frequency of $200 \mathrm{~Hz}$ was used for both systems. Gait events were detected for OMC outcomes using a multi-component (three-plate) time-synchronized, floor-embedded force platform (Model FP9060-15, Bertec Corporation) (1000 Hz sampling frequency) and for the IMU system using an IMU-based algorithm provided by the commercial software (MyoRESEARCH 3.10.64 [MR3]). OMC markers and IMUs were placed on the participant simultaneously to enable 
concurrent measurements by both systems. A single rater (motion capture trained physiotherapist) placed 22 passive retro-reflective markers (14 mm diameter) on anatomical landmarks according to a validated modified lower body CGM model (Plug-in-Gait [PiG]) provided by the OMC software, Nexus 1.8 .5 (calcaneus; medial and lateral malleoli; second metatarsals; shanks [using wands, along the alignment of the ankle flexion axis]; tibial tuberosities; lateral and medial knees; lateral thighs; anterior superior iliac spines; and posterior superior iliac spines). Markers were not removed during any trials and thus could not introduce placement bias. For the rigid lower body model setup of the IMU system, IMUs were placed on the pelvis (posteriorly on the sacral surface), thighs (lateral attachment to the lower quadrant of the segment, i.e. the area of lowest muscle belly displacement during walking), shanks (anteromedial surface of the tibia), and feet (dorsally and sufficiently proximal to the equinus break to avoid excessive IMU motion). IMUs were attached with a fixation strap (pelvis), elasticated Velcro straps (thighs and shanks) and double-sided tape, firmly reinforced using elastic adhesive bandage (feet). These placements were done in such a way that no movement restrictions were imposed. All markers/IMUs were checked by the first author and laboratory technician throughout measurement procedures to ensure that they remained in place. Fig. 1[A] shows concurrent marker and IMU placement.

\subsection{Practice trials}

Participants were explicitly instructed to walk "as normal as possible". To familiarize participants to the feeling of walking with the fixated IMU/markers, practice trials were performed along the capture volume, noting specific starting positions for optimal force plate foot strikes, and until the rater was satisfied that the participant assumed a relaxed, normal gait (approximately 2-3 practice trials per participant). To avoid any potential force plate targeting, participants were instructed to look ahead and focus on the laboratory wall in front at eye level.

\subsection{Model calibration}

\subsubsection{OMC system/model}

Anthropometrics (leg lengths, ankle- and knee widths, height) were measured as required for the CGM (OMC model). A static anatomical CGM calibration with the participant standing on the force plate was performed according to standard laboratory protocol. This was done once-off per participant and prior to IMU calibration; therefore, ferromagnetic interferences were not a concern.

\subsubsection{IMU system/model}

The IMU model was calibrated as per the user guide by having the participant remain stationary in a neutral reference posture during calibration (Fig. 1[A]), to establish the local coordinate system and provide a reference angle on which to base the kinematic data. Calibrations were performed on a $30 \mathrm{~cm}$-high wooden platform, minimizing exposure to potential floor-based magnetic distortions. Substantial attention was paid to postural setup to minimize subsequent measurement errors. The rater provided standardized instructions and inspected the calibration pose in three planes, enforcing neutral joint/segment angles. Standardized instructions were to (1) place feet parallel and facing forward, (2) face the pelvis directly forward in the same direction as the feet, (3) vertically align hip-, knee- and ankle joint centers (to position feet hip-width apart) and (3) stand straight up with arms crossed. IMU calibration was then initiated. Participants were instructed to remain static until completion of the $\sim 15$ s system calibration (indicated by an audio signal). The IMU system allows calibration data to be used for repeated measure series of data collection; however, frequent re-calibration of sensors within test series is recommended by the manufacturer to avoid drift over time. Thus, the system was re-calibrated each time directly before a gait trial.

\subsection{Data collection}

Participants performed a static IMU calibration pose on the wooden platform, thereafter walked barefoot at habitual speed along a $10-\mathrm{m}$ level walkway, and immediately returned to the wooden platform. Six repeated trials were performed successively and in the same direction each time (one trial = calibration pose followed directly by a walk). A gait trial was deemed successful if the entire landing foot contacted at least one force plate and if participants did not target the force plate by looking down at it or by noticeably changing stride length.
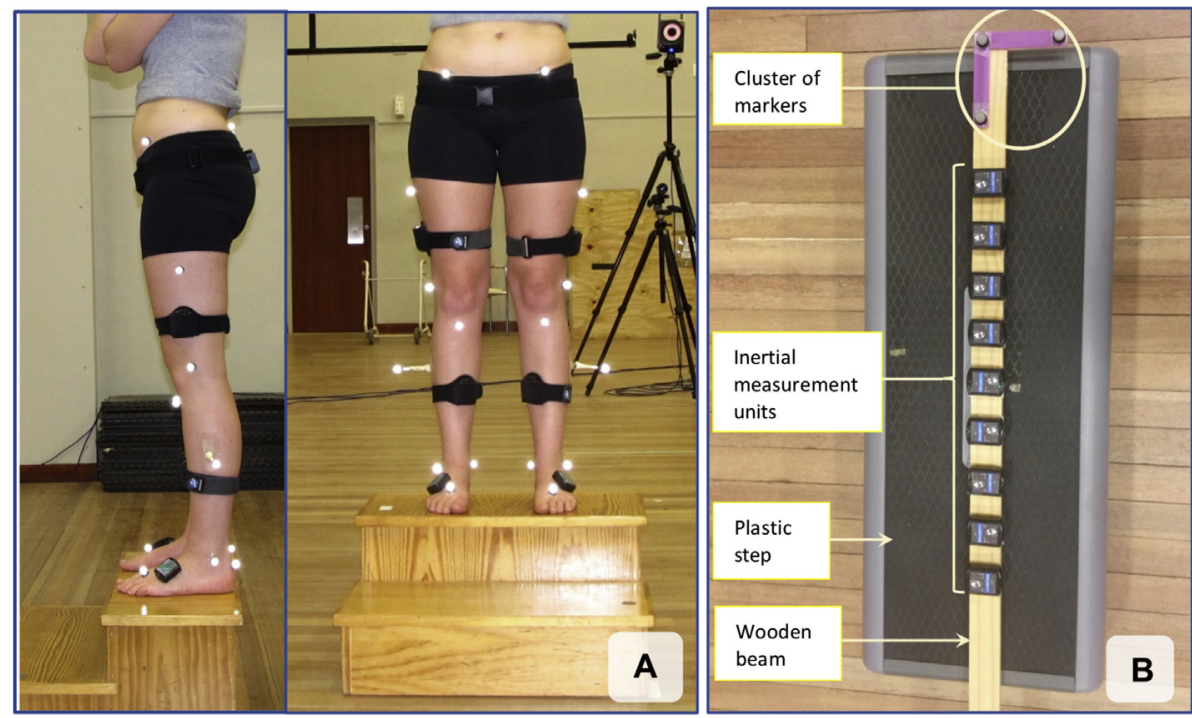

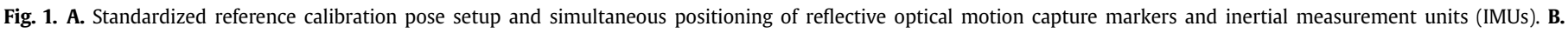
Experimental setup for testing static and dynamic IMU tracking. 
The IMU calibration poses were recorded using OMC to measure actual anatomical angles. IMU postural measurements are not available to the system during its own calibration. The IMU system assumes joint/segment angular values of zero in all planes during calibration and zero-values were thus used for later comparative analysis.

\subsubsection{IMU orientation tracking}

Static and dynamic IMU orientation tracking accuracy was assessed in a separate experiment to the clinical outcomes (onceoff for the entire study). A wooden beam was rigidly mounted with nine IMUs and three OMC markers (Fig. 1[B]). To determine relative orientation of OMC and IMU global coordinate systems, a doublepose method was applied (Faber et al., 2013). IMU and OMC local frames were subsequently aligned by initializing them to an orientation of unity. The static trial was collected with the beam resting passively on a raised plastic step for $60 \mathrm{~min}$ (long enough to detect gyroscope drift error). In a separate 5-min trial, following Kalman filter reset, concurrent IMU and OMC measurements were taken during sustained motion of the beam at angular velocities exceeding those of lower limb segments during gait (enough to detect motion-induced tracking errors).

\subsection{Data processing}

\subsubsection{OMC system $/$ model}

Pre-processing of OMC gait trials was done in Nexus software. Marker trajectories were reconstructed and labelled using standard Nexus functions, then smoothed with a fourth-order, zero-lag lowpass Butterworth filter (6 Hz cut-off) (Yu et al., 1999). Joint and segment kinematics were calculated from the CGM model using the standard dynamic PiG pipeline, which determines hip joint centers using the Davis equations (Davis et al., 1991). Knee axis estimation was performed by optimizing the thigh-rotation offset parameter during gait (Schache et al., 2006), and ankle axis estimation by determining the shank-rotation offset parameter during the static trial using medial and lateral malleolus markers. OMC gait events were detected from force plate data; initial contact was determined at the first frame where the vertical ground reaction force exceeded $20 \mathrm{~N}$, and toe-off was calculated at the first frame of less than $20 \mathrm{~N}$ (a standard method in gait analysis for avoiding false detections due to high frequency measurement noise, which can be up to approximately $20 \mathrm{~N}$ ).

\subsubsection{IMU system/model}

The IMU biomechanical model that provided joint and segment kinematics was included in the commercial software (MR3) and complies with International Society of Biomechanics (ISB) recommendations for lower limb joints (Wu et al., 2002). Motion-related signals were transmitted wirelessly to the IMU software on a recording laptop. The software automatically filters raw data using a robust fusion algorithm (Kalman filter) optimized for IMU data. IMU angular orientations are estimated at IMU-level. Thus, data pre-processing was not needed, but data were corrected for magnetic drift (distortion) on the foot, shank and thigh segments. A fourth-order zero-lag low pass Butterworth filter $(6 \mathrm{~Hz})$ was used to filter IMU data in MR3. The commercial software's contact detection algorithm also outputs a contact signal for IMU data, utilizing foot angular velocity and acceleration measurements from the footIMU. Initial foot-contact and foot-off events were calculated from each foot-contact signal.

Data recorded in the IMU and OMC software were respectively exported to single .csv and .c3d files and imported into MATLAB software (R2017a, MathWorks). For both OMC and IMUs, gait cycle time-normalization (101 data points at time intervals of $1 \%$ ) and data collation were performed using custom-built scripts in MATLAB. In cases where a gait trial contained more than one complete and valid gait cycle for one or both legs, only the gait cycle for each leg judged to contain the best data quality was retained for analysis.

\subsubsection{Offset adjustments of IMU output}

A second IMU dataset was created wherein IMU-measured gait kinematics were adjusted by the OMC-measured Euler angle offset (i.e. offset measured by OMC during IMU calibration trials). This was done to enable IMU-OMC comparisons without the presence of biomechanical modelling differences (i.e. a model adjusted comparison). While this approach is less accurate than using matrix mathematics to reorient anatomical frames of the IMU model using OMC data, the matrix approach would not have allowed for an evaluation of the commercial IMU model and calibration, which were being validated for this study. The Euler angle approach can be considered conservative for validation purposes, as it would presumably remove model differences less effectively. Therefore, if the Euler angle offset method was to produce sufficiently valid IMU comparison results, the matrix approach would most likely have produced slightly better (not worse) results.

Since time shifts in the gait cycle data (due to differences in gait event detection and thus segmentation) could lead to comparison errors, a third IMU dataset was created wherein the model adjusted gait kinematics were further adjusted for time-segmentation differences due to between-system gait event detection differences (i.e. model-and-time adjusted IMU output). IMU time-series data from the biomechanical model were time-synchronized to OMC data offline by aligning a common event (the peak knee flexion angle). The IMU data were thereafter segmented and timenormalized using OMC events to allow for comparison of the systems' data independent of event detection error.

\subsection{Data analysis}

Six calibration poses and six walking strides (one valid mid-walk stride from each of the calibrated trials) were analyzed per participant to mitigate the contribution of intra-individual performance variability (Monaghan et al., 2007). This resulted in 90 strides for analysis. Ensemble means of angular curves were calculated for each participant, system and joint/segment (averaged for each participant across trials, and averaged across participants) and used for further analysis. For gait, secondary knee and ankle angles (frontal and transverse planes; e.g. rotation, in/eversion, varus/valgus) were not analyzed as these outcomes are less reliable even using OMC, hampering clinical interpretation (Della Croce et al., 2005). For the calibration poses, joint angles were compared instead of segment orientations, because angles constituted the criteria being inspected visually during setup. End-point segments (pelvis and feet) were compared in the sagittal and coronal planes. However, the direction participants faced was not strictly controlled/assumed during IMU calibration, but rather inferred by the system measurements. Therefore, validity of the transverse plane pelvis and feet orientations were assessed relative to each other, since a shared heading is assumed by the IMU calibration.

\subsection{Statistical analysis}

Analyses were performed in SPSS (Version 25.0) and MATLAB. Clinical significance was considered as differences $>5^{\circ}$ (McGinley et al., 2009) and statistical significance as $p<0.05$. Concurrent validity was assessed as waveform dissimilarity and agreement among the joint angle time histories. Comparisons were performing for unadjusted (direct), model adjusted and model-and-time 
adjusted IMU output versus OMC/CGM. For dissimilarity, IMU and OMC curves' relative means were used to align the curves, and rootmean-square error (RMSE) (averaging over one gait cycle) was calculated. For agreement, Bland-Altman analyses were performed, including 95\% limits of agreement (LoA). Linear association of gait curves were additionally assessed by calculating Pearson's correlation coefficients (r); interpreted according to Evans (1996).

Within-session reliability of gait curves were quantified using SEM. For each kinematic outcome, a two-way repeated-measure analysis of variance (ANOVA) was performed. SEM was calculated as the square root of the mean-square-error $\left(\mathrm{MS}_{\mathrm{E}}\right)$ from the ANOVA: SEM $=\sqrt{\mathrm{MS}_{\mathrm{E}}}$. This approach is robust to betweenparticipant variability and thus preferable to calculations based on the intraclass correlation (Hopkins, 2000; Weir, 2005). The upper $95 \%$ confidence limit (CL) of the SEM was calculated using a sample-and-trial-specific multiplying factor of 1.2 (Hopkins, 2000).

For the calibration poses, intra-rater repeatability was assessed using SEM. Calibration pose accuracy was based on the mean absolute difference (MAD) of the achieved angles (quantified by OMC) and anatomical zero. Finally, IMU tracking accuracy was determined using RMSE, to demonstrate dissimilarity between internally-measured (by IMU) and externally-measured (by OMC) IMU orientation.

\section{Results}

\subsection{IMU concurrent validity (gait)}

\subsubsection{Unadjusted output}

For unadjusted (direct) comparisons, only hip abduction/ adduction and pelvic rotation and -obliquity RMSE were $<5^{\circ}$ (Fig. 2, also Additional File 1). Pelvic tilt and hip flexion/extension demonstrated the highest RMSE (largest dissimilarity) and pelvic rotation the lowest. Bland-Altman (Fig. 3) revealed clinically acceptable agreement (biases $\leq 4.2^{\circ}$ ), except for sagittal pelvic, hip and ankle angles $\left(>5^{\circ}\right)$. LoA were $<5^{\circ}$ for all secondary angles (except hip rotation); while all sagittal LoA exceeded $5^{\circ}$.

Unadjusted gait curves correlated very strongly $(r>0.80)$ to OMC-derived curves for sagittal plane ankle, hip and knee signals, hip abduction/adduction and pelvic rotation. Hip rotation demonstrated the weakest correlation (Table 1).

\subsubsection{Model adjusted output}

All model adjusted angles (except hip rotation) demonstrated clinically acceptable concurrent validity (RMSE $<5^{\circ}$ ). Further time adjustments did not produce statistically significant changes in RMSE; except for knee flexion/extension (a statistically, but not clinically, significant difference of $1.6^{\circ}, \mathrm{p}<0.001$ ). Fig. 2 illustrates the RMSE for model-and-time adjusted gait outcomes versus unadjusted results. Full data are presented in Additional File 1. Modeland-time adjustments demonstrated clinically acceptable agreement for all outcomes: maximum bias of $4.3^{\circ}$ (pelvic tilt); all other biases $\leq 1.6^{\circ}$. All calibration-and-time offset adjusted LoA were $<$ $5^{\circ}$, except for pelvic tilt (upper LoA: $11.1^{\circ}$ ) (Fig. 3). Model-and-time adjusted IMU gait curves demonstrated similar correlations to OMC as unadjusted output (Table 1 ).

\subsection{IMU within-session reliability (gait)}

SEM values for gait angles were clinically acceptable and ranged between $1.1^{\circ}$ and $3.4^{\circ}$. These values were generally $<2^{\circ}$, except for knee flexion/extension, hip rotation and pelvic rotation (Fig. 2, also Additional File 1).

\section{Dissimilarity between IMU- and OMC-measured gait angles}

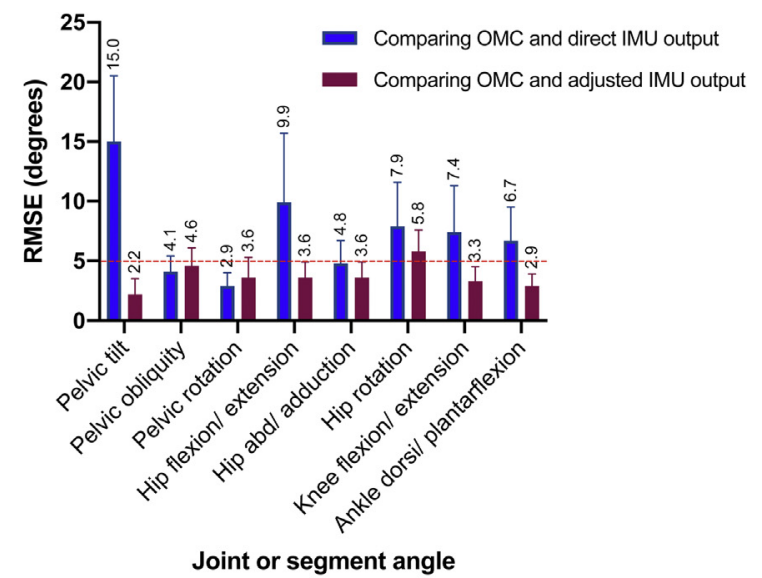

Within-session reliability for IMU-measured gait angles

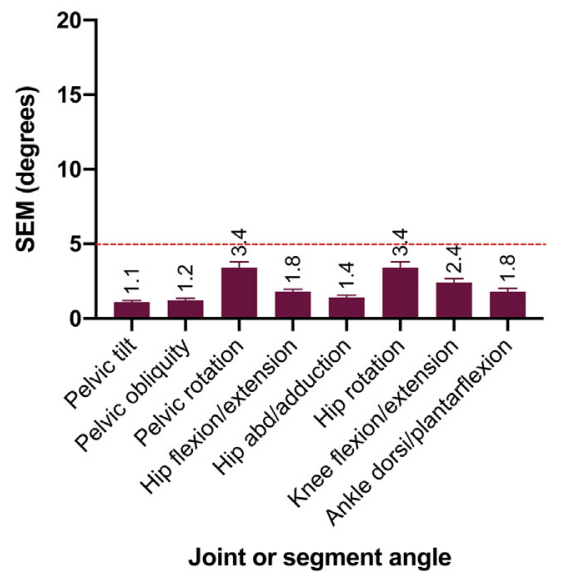

Fig. 2. Results for IMU-measured gait relative to a minimum clinically important difference (MCID) of $5^{\circ}$. The top graph shows angular dissimilarity between OMC and IMU systems as the root-mean-square error (RMSE) for unadjusted and adjusted (calibration-and-time) comparisons. The bottom graph shows within-session reliability of IMU-measured gait kinematics as the standard error of measurement (SEM) and upper 95\% confidence limits (error bars).

\subsection{Calibration pose accuracy}

Calibration pose accuracy was clinically acceptable $\left(\mathrm{MAD}<5^{\circ}\right)$ for all angles except pelvic tilt, hip flexion, knee rotation and ankle dorsiflexion (Fig. 4, also Additional File 2). Low standard deviations (SD) confirmed systematic offsets for ankle dorsiflexion $\left(2.6^{\circ}\right)$ and knee rotation $\left(4.8^{\circ}\right)$. Although knee extension demonstrated a low $\operatorname{MAD}\left(1.3^{\circ}\right)$, its SD indicated clinically significant random error $\left(5.5^{\circ}\right)$ due to an outlier $\left(13.9^{\circ}\right)$.

\subsection{Calibration pose repeatability}

Calibration pose repeatability was clinically acceptable (SEM $<2.2^{\circ}$, Fig. 4 , also Additional File 2$)$. Knee rotation showed the highest upper $95 \% \mathrm{CL}\left(2.6^{\circ}\right)$.

\subsection{IMU tracking accuracy}

IMU tracking was accurate. Static IMU tracking RMSEs were $0.4^{\circ}$ $\pm 0.2^{\circ}$ (inclination) and $0.8^{\circ} \pm 0.4^{\circ}$ (heading). Dynamic tracking RMSEs were $0.9^{\circ} \pm 0.2^{\circ}$ (inclination) and $2.0^{\circ} \pm 0.8^{\circ}$ (heading). 
Pelvic tilt (direct IMU output)

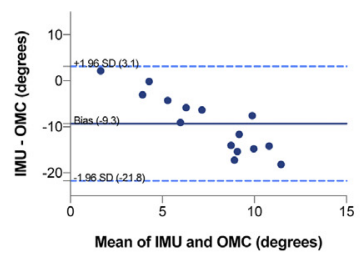

Pelvic rotation (direct IMU output)

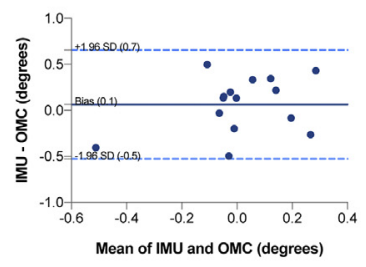

Hip ab/adduction (direct IMU output)

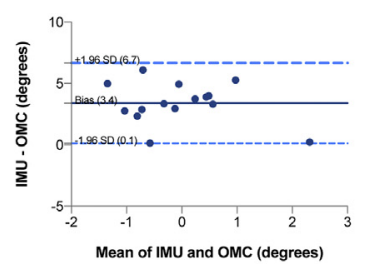

Knee flexion/extension (direct IMU output)

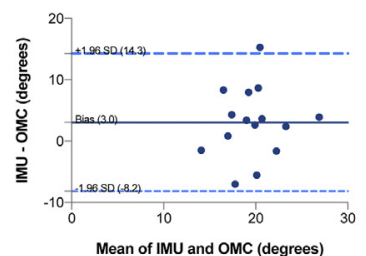

Pelvic tilt (adjusted IMU output)

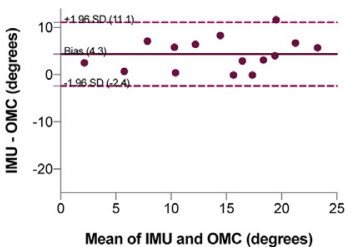

Pelvic rotation (adjusted IMU output)

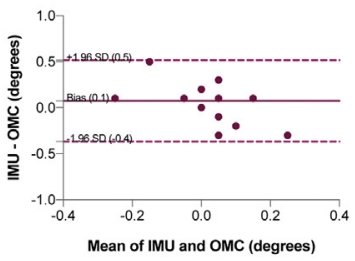

Hip ab/adduction (adjusted IMU output)

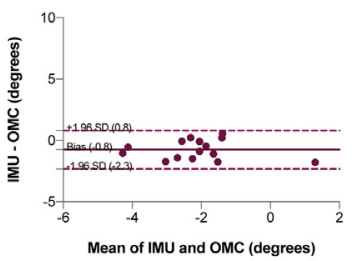

Knee flexion/extension (adjusted IMU output)

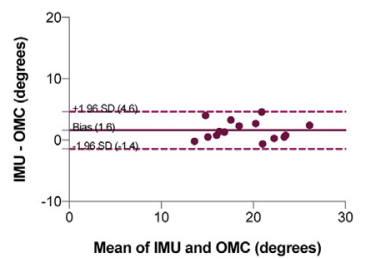

Pelvic obliquity (direct IMU output)

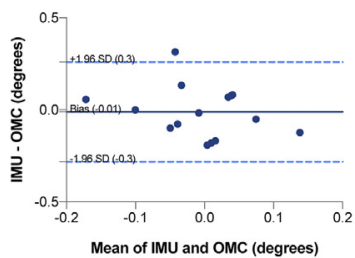

Hip flexion/extension (direct IMU output)

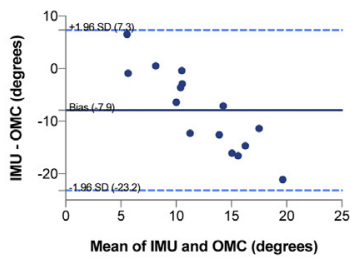

Hip rotation (direct IMU output)

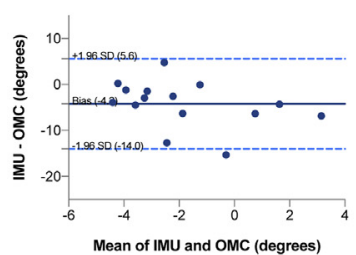

Ankle dorsi/plantarflexion (direct IMU output)

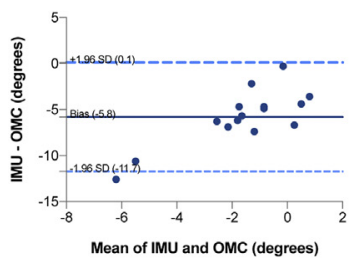

Pelvic obliquity (adjusted IMU output)

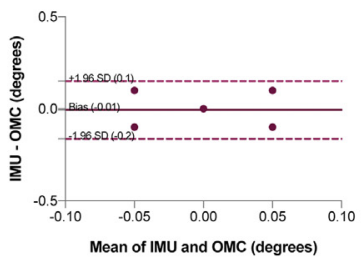

Hip flexion/extension (adjusted IMU output)

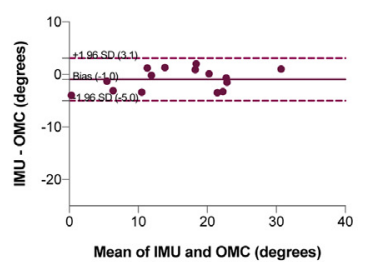

Hip rotation (adjusted IMU output)

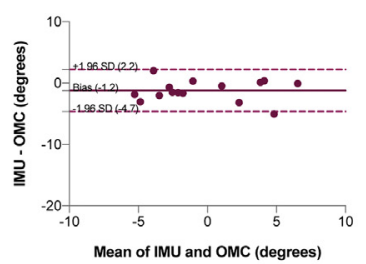

Ankle dorsi/plantarflexion (adjusted IMU output)

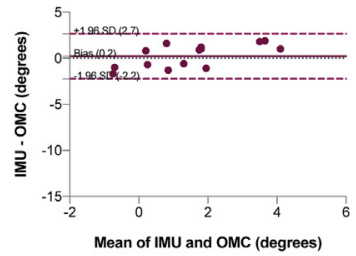

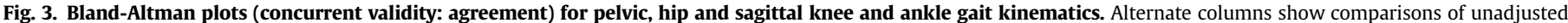

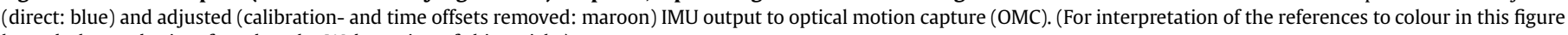
legend, the reader is referred to the Web version of this article.)

Table 1

Linear correlation (Pearson's $\mathrm{r}$ ) for unadjusted comparisons and model adjusted comparisons of IMU-measured gait angles. Optical motion capture served as reference standard.

\begin{tabular}{|c|c|c|c|c|}
\hline \multirow[t]{2}{*}{ Joint or segment angle } & \multicolumn{4}{|c|}{ Concurrent validity: Linear correlation (r) } \\
\hline & $r_{\text {unadjusted }}$ & $r$ interpretation & $\mathrm{r}_{\mathrm{cal}+\text { time }}$ & $\mathrm{r}$ interpretation \\
\hline Pelvic tilt & 0.58 & Moderate & 0.60 & Strong \\
\hline Pelvic obliquity & 0.48 & Moderate & 0.38 & Weak \\
\hline Pelvic rotation & 0.92 & Very strong & 0.94 & Very strong \\
\hline Hip flexion/extension & 0.99 & Very strong & 0.99 & Very strong \\
\hline Hip abd/adduction & 0.87 & Very strong & 0.82 & Very strong \\
\hline Hip rotation & 0.44 & Moderate & 0.40 & Moderate \\
\hline Knee flexion/extension & 0.97 & Very strong & 0.99 & Very strong \\
\hline Ankle dorsi/plantarflexion & 0.93 & Very strong & 0.97 & Very strong \\
\hline
\end{tabular}

Abbreviations: IMU = inertial measurement unit.

Model adjusted values shown for full adjustment (time and calibration offsets removed).

Correlations of $0.20 \leq 0.39$ were deemed weak; $0.40 \leq 0.59$ were considered moderate; $0.60 \leq 0.79$ were considered strong; and $0.80 \leq 1.00$ very strong.

\section{Discussion}

The primary aim of this study was to assess the concurrent validity (versus OMC/CGM) and within-session reliability of lower limb gait kinematics measured by a frequently calibrated commercial IMU system. Results revealed that between-system comparisons yielded clinically significant differences $\left(>5^{\circ}\right)$ for all sagittal angles and hip rotation, but after accounting for modelling differences, between-system differences for all angles (except hip rotation) were clinically acceptable $\left(<5^{\circ}\right)$. Secondly, IMU- and 
IMU calibration pose accuracy

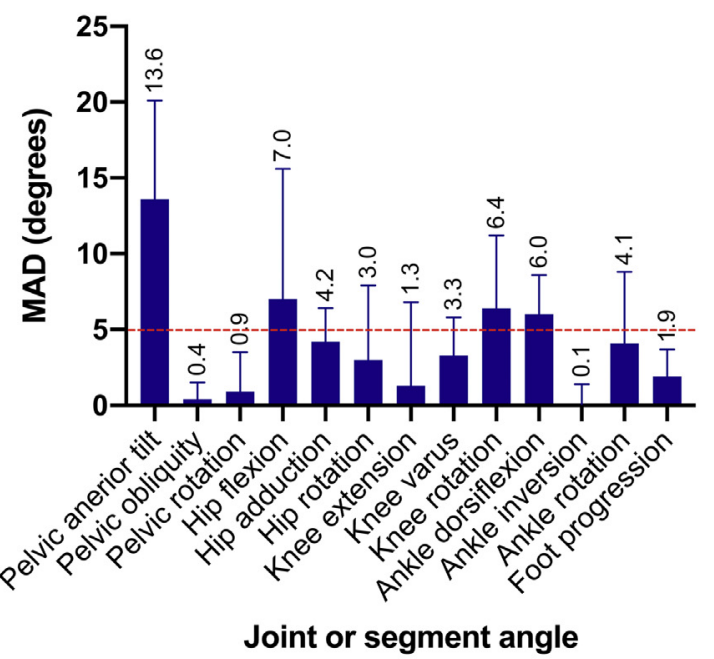

IMU calibration pose intra-rater repeatability

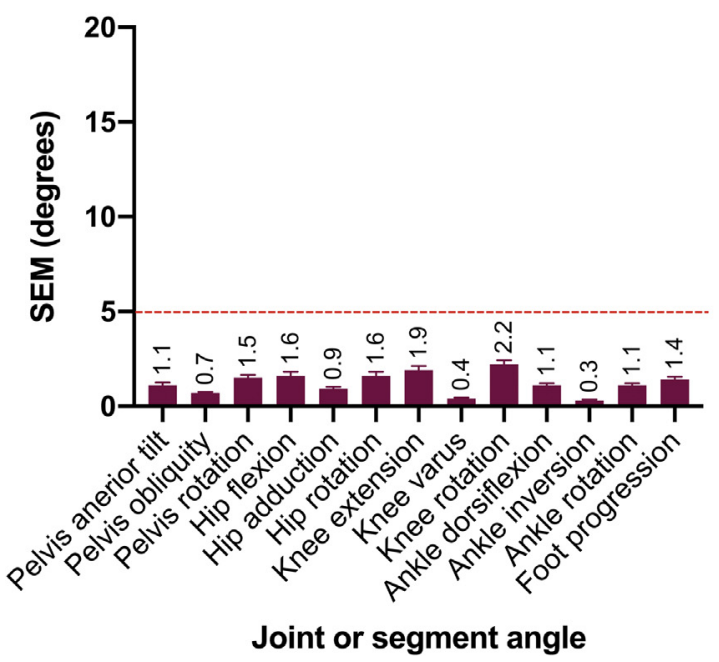

Fig. 4. Results for IMU reference calibration pose relative to a minimum clinically important difference (MCID) of $\mathbf{5}^{\circ}$. The top graph shows accuracy as average optical motion capture (OMC)-measured static angles (mean absolute difference [MAD] from zero). The bottom graph shows intra-rater repeatability as standard error of measurement (SEM) and upper 95\% confidence limits (error bars).

OMC-measured gait curves correlated highly for all angles except hip rotation, pelvic tilt and pelvic obliquity. Thirdly, IMU-measured gait angles demonstrated clinically acceptable within-session reliability $\left(<5^{\circ}\right)$ for all measured angles, with measurement error falling below $2^{\circ}$ for all angles except pelvic- and hip rotation. Low concurrent validity for unadjusted gait comparisons indicates that results are not directly comparable between systems, but good reliability and strong correlation to OMC imply that IMU gait data are comparable within, and possible between, participants when using the same system. In terms of the secondary study aims, results showed that an IMU calibration pose can be implemented accurately and repeatably by a trained user for most investigated angles, and that IMU tracking was accurate. A key observation regarding calibration setup was that while differences in hip flexion reflected expected differences in pelvic tilt, ankle dorsiflexion showed a currently undocumented offset; inherent to maintaining postural balance with straight knees.
A previous study (Seidel et al., 2015) using the same IMU system as this investigation (myoMOTION) compared gait measurements to OMC and reported RMSE values for sagittal ankle, knee and hip angles of $8.2^{\circ}, 5.2^{\circ}$ and $8.4^{\circ}$ respectively (pelvis not investigated). These values are similar to this study's unadjusted results (Fig. 2), and the use of a different reference OMC system (Qualisys) may explain subtle discrepancies. Similar to Seidel et al. (2015), who demonstrated r-values of $0.73-0.98$ for sagittal plane hip, knee and ankle and frontal plane hip kinematics, the current study also demonstrated high between-system correlations (regardless of accounting for modelling differences). Notably, angles considered as primary gait drivers (sagittal plane hip, knee and ankle) demonstrated the highest correlations (close to 1). The high RMSE observed in the present study for hip rotation (not assessed by Seidel et al., [2015]), despite adjustment, may largely stem from factors such as soft tissue artefact (STA) (Chiari et al., 2005); and the high RMSE for unadjusted pelvic tilt from calibration offset due to anatomical pelvic position. Conservative removal of calibration/ modelling differences improved all RMSE for gait (except hip rotation) to $<5^{\circ}$. All angles demonstrated good consistency across trials $\left(\mathrm{SD}<2^{\circ}\right.$ ); agreeing with reported gait analysis errors (except for hip and knee rotation) (McGinley et al., 2009). The additional low correlations and high RMSE observed in the present study for hip rotation, pelvic obliquity and pelvic tilt, despite modelling adjustment, may be explained by low signal-to-noise ratios (Shull et al., 2014). Joints and segments with small ranges of motion have largely stationary signals, and much of the observed movement may be noise caused by factors such as STA.

Unadjusted comparisons further revealed clinically significant sagittal plane pelvic, hip and ankle biases. However, after accounting for modelling differences, biases were $<5^{\circ}$, as were all LoA (except pelvic tilt). SEM values for IMU-measured gait angles suggest clinically acceptable reliability; agreeing with reports for OMC in healthy adults (e.g. SEM $<2^{\circ}$ (Wilken et al., 2012); and generally $<4^{\circ}$ for sagittal, and $\sim 2^{\circ}$ for coronal angles (McGinley et al., 2009)). Together, results indicate that accepting a measurement error of $5^{\circ}$ (McGinley et al., 2009) is reasonable for lower limb angles (except hip rotation) when using the commercial IMU system implemented in this study, although this may differ for gross gait pathology. Knowledge of a system's measurement error provides greater confidence that observed differences, or values exceeding these, are real (Kavanagh and Menz, 2008). This IMU system's good consistency lessens the risk of excessive noise drowning out real effects.

The calibration pose was investigated as an innate contributor to IMU gait validity. Accurate calibration poses are important to establish anatomically interpretable joint/segment angles. Although studies have assessed the reliability of such poses, few have evaluated accuracy. Robert-Lachaine et al. (2017) were among the few that also assessed accuracy by defining a "perfect" poseexecution using $0^{\circ}$-criteria. These authors assessed passivelyplaced neutral-pose accuracy using OMC as reference, demonstrating pelvic tilt offset exceeding $10^{\circ}-$ similar to the current study's results. The current results also agree with clinically reported anterior pelvic tilt means of $11^{\circ}-13^{\circ}$ (range: $0^{\circ}-23^{\circ}$ ) during normal stance in able-bodied adults (Kroll et al., 2000; Levine and Whittle, 1996; Preece et al., 2008). In humans, standing with $0^{\circ}$ pelvic tilt, $0^{\circ}$ hip flexion/extension and $0^{\circ}$ ankle dorsiflexion/ plantarflexion would displace the center of mass (COM) posteriorly and undermine balance. Pelvic tilt offset was confirmed to be reflected in hip flexion offsets - a well-documented bias - but also noted consistent dorsiflexion offset: a novel observation. This is explained by considering that the COM (and thus hip joint center) is typically positioned above the midfoot (anterior to the ankle) to ensure postural stability. Therefore, if the hip joint center is anterior 
to the ankle, with straight knees, the ankle must be dorsiflexed. Only for sagittal knee, hip and pelvic accuracy did random error exceed $5^{\circ}$; possibly due to marker errors. Actual inter-participant variation may further explain pelvic and hip offset, considering the wide range of pelvic tilt angles reported for adults. Quadriceps angle (Q-angle) variability may explain hip and knee coronal offsets in the current study's all-female sample. Women reportedly have $3.4^{\circ}-4.9^{\circ}$ higher Q-angles than men (Conley et al., 2007). Coronal pelvic offsets were lower than the hip, indicating femoral angulation; further reflected by relatively higher knee-versus-ankle coronal offsets. Good accuracy was observed regarding parallel foot and pelvic positioning. The inter-participant random error noted for pelvic rotation may be due to pelvic marker placement error (Hara et al., 2014) rather than faulty foot positioning. Overall, IMU calibration pose implementation was accurate, considering that key setup angle errors were negligible, systematic offsets were anatomically plausible, and random errors mostly $<5^{\circ}$.

Similar to the current study's findings, good repeatability is reported for IMU calibration poses, trending towards transverse angles being less so (Picerno, 2017; Picerno et al., 2008; RobertLachaine et al., 2017). Proper comparisons to the literature are however hampered by heterogeneous published protocols and outcomes. Robert-Lachaine et al. (2017) reported SEM for a passively-placed neutral calibration pose of $0.9^{\circ}-1.8^{\circ}$ (sagittal), $0.5^{\circ}-1.3^{\circ}$ (coronal) and $0.8^{\circ}-3.2^{\circ}$ (transverse); mirroring the current study's results.

In the present study, accounting for modelling differences by removal of calibration offset values rendered the composite between-system error for gait data generally below $5^{\circ}$. Further time-offset adjustments did not yield clinically significant changes, confirming the larger contribution of calibration offsets (rather than event detection). Remaining differences from the CGM likely comprise combined calibration variability, STA and IMU tracking errors. Regarding IMU orientation tracking, this study independently demonstrated accurate results ( $\leq 2^{\circ}$ error). Since at least two IMUs are involved in joint angle estimation, joint tracking errors of up to $\sim 4^{\circ}$ are theoretically plausible (although these errors cannot necessarily be summed linearly). STA errors may also contribute substantially to the observed composite betweensystem errors between systems (especially for hip rotation); considering STA values reported for the thigh (high: $12^{\circ}-15^{\circ}$ ), foot (moderate: $2^{\circ}-6^{\circ}$ ) and shank (small: $0.3^{\circ}-1.0^{\circ}$ ) (Peters et al., 2010).

\subsection{Study limitations}

Study limitations include the all-female sample. However, recent empirical studies on gait kinematics show that while sex may influence coronal kinematics (increased pelvic obliquity being the most consistent observation) (Bruening et al., 2015; Frimenko and Whitehead, 2014), consistent correlations between sex and sagittal/transverse kinematics are not reported for usual-paced gait (Chehab et al., 2017; Frimenko and Whitehead, 2014). The current study findings are not generalizable to gross gait pathology or above-normal BMI. Although OMC/CGM served as reference, it is susceptible to faulty marker placement (Della Croce et al., 2005) and STA (Leardini et al., 2005); nevertheless, marker-placement by a laboratory-trained physiotherapist, and normal participant BMIs, likely limited these. Although IMU calibration pose test-retest and inter-rater reliability were not investigated, reliability in terms of repeated IMU-placement probably at least matches OMC-markerplacement reliability, since IMUs require no accurate placements. It should further be noted that although a conservative bias removal approach was adopted, this study illustrated that differences in system outputs are largely due to calibration offsets and not IMU tracking (removing calibration offsets rendered errors < $5^{\circ}$ ). Finally: not controlling for STA introduces a methodological bias. However, it may be argued that it is redundant to differentiate STA from calibration errors for very small dynamic differences, as the combined STA- and calibration error was already $<5^{\circ}$.

\subsection{Conclusion}

This study demonstrated that with frequent calibration, a commercial IMU-based system provides clinically acceptable estimation of lower limb gait angles in normal-weight, healthy female adults. IMU gait data should however not be compared directly to systems using other models. IMU-measured gait angles are reliable, and a measurement error of $5^{\circ}$ may be accepted for this system. A trained rater can accurately and repeatably implement the calibration poses required by the system, although observations were joint/segment-specific.

\subsection{Clinical relevance}

- IMU-based systems are significantly more portable and affordable than OMC systems, are commercially available and thus offer researchers and clinicians an important tool for collecting biomechanical data outside traditional laboratory spaces. This study shows that IMU-measured gait data are clinically useful and comparable within (possibly between) participants when using the same system.

- Frequent re-calibration of IMUs between gait trials is recommended by manufacturers to avoid drift errors accumulating over time. Calibration procedures are however among the important error sources affecting IMU gait measurements. This study shows that using standardized instructions, a clinician can repeatably and accurately set up a simple, single-pose reference calibration posture in-between gait trials, and that subsequent gait trials remain reliable.

- Despite good correlations existing between IMU- and OMCmeasured gait data, a clinical gait analysis conducted using IMUs cannot be compared to OMC datasets (including normative OMC-measured gait data from the literature or a gait laboratory). Comparative datasets for interpreting IMU data should be established using the same system/model.

- Study results suggest that clinicians may accept a measurement error of $5^{\circ}$ when using this specific IMU system in normalweight women without gross gait pathology (except for hip rotation, for which at least $6^{\circ}$ may be accepted).

\section{Funding}

Research reported in this publication was supported by the South African Medical Research Council under a Self-Initiated Research Grant, as well as by the Harry Crossley Foundation (Grant numbers SU-PT-15/10-000005 and SU-PT-16/000057). The PhD from which this study emanated was funded by the South African Medical Research Council in terms of the National Health Scholars Programme from funds provided for this purpose by the National Department of Health, South Africa. The views and opinions expressed are not those of the funders, but of the authors of the material publicized. The study sponsors had no role in the study design, in the collection, analysis and interpretation of data; in the writing of the manuscript; and in the decision to submit the manuscript for publication.

\section{Ethics approval}

This study was conducted according to the Declaration of Helsinki. Ethical approval from the Stellenbosch University (SU) Health 
Research Ethics Committee (HREC) was granted (N15/05/043) and the SU Division of Institutional Research and Planning provided additional permission for including staff and students as participants. Written informed consent was obtained from all participants prior to data collection.

\section{Availability of data and supporting materials}

The data sets generated and analyzed in this study are either presented in the Additional Files or available from the corresponding author on reasonable request.

\section{CRediT authorship contribution statement}

Karina Berner: Conceptualization, Data curation, Formal analysis, Funding acquisition, Investigation, Methodology, Project administration, Resources, Validation, Visualization, Writing original draft. John Cockcroft: Conceptualization, Data curation, Formal analysis, Investigation, Methodology, Resources, Software, Validation, Writing - review \& editing. Linzette D. Morris: Conceptualization, Methodology, Supervision, Writing - review \& editing. Quinette Louw: Conceptualization, Funding acquisition, Methodology, Project administration, Resources, Supervision, Writing - review \& editing.

\section{Declaration of competing interest}

$\mathrm{KB}, \mathrm{LDM}$ and $\mathrm{QL}$ confirm that they have no conflicts of interest related to this manuscript. JC is currently a paid consultant to Noraxon USA Inc. However, the present study was conducted entirely independent from Noraxon as part of a PhD project by KB. Noraxon did not provide any funding, incentives, or services for any aspect of this study; nor did the company play any role in the study design, data collection, analysis and interpretation; in the writing of the manuscript; or in the decision to submit the manuscript for publication.

\section{Acknowledgements}

We thank all study participants for their patience and enthusiasm, the South African Medical Research Council and Harry Crossley Foundation for financial support and Stellenbosch University for the use of their facilities and relevant staff.

\section{Appendix A. Supplementary data}

Supplementary data to this article can be found online at https://doi.org/10.1016/j.jbmt.2020.06.008.

\section{References}

Ali, A, Sundaraj, K., Ahmad, B., Ahamed, N., Islam, A, 2012. Gait disorder rehabilitation using vision and non-vision based sensors: a systematic review. Bosn. J. Basic Med. Sci. 12, 193-202.

Ancillao, A., 2016. Analysis and measurement of human motion: modern protocols and clinical considerations. J Robot Mech Eng Res 1, 30-37.

Astephen, J.L., Deluzio, K.J., Caldwell, G.E., Dunbar, M.J., 2008. Biomechanical changes at the hip, knee, and ankle joints during gait are associated with knee osteoarthritis severity. J. Orthop. Res. 26, 332-341. https://doi.org/10.1002/ jor.20496.

Balasubramanian, S., 2013. Comparison of angle measurements between Vicon and MyoMotion systems. In: http://www.noraxon.com/?smd_process_ download $=1 \&$ download id $=6949$.

Begg, R.K., Sparrow, W.A., 2006. Ageing effects on knee and ankle joint angles at key events and phases of the gait cycle. J. Med. Eng. Technol. 30, 382-389.

Bruening, D.A., Frimenko, R.E., Goodyear, C.D., Bowden, D.R., Fullenkamp, A.M., 2015. Sex differences in whole body gait kinematics at preferred speeds. Gait Posture 41, 540-545. https://doi.org/10.1016/J.GAITPOST.2014.12.011.

Chehab, E.F., Andriacchi, T.P., Favre, J., 2017. Speed, age, sex, and body mass index provide a rigorous basis for comparing the kinematic and kinetic profiles of the lower extremity during walking. J. Biomech. 58, 11-20.

Chen, S., Lach, J., Lo, B., Yang, G.-Z., 2016. Toward pervasive gait analysis with wearable sensors: a systematic review. IEEE J Biomed Heal Informatics 20, 1521-1537.

Chiari, L., Della Croce, U., Leardini, A., Cappozzo, A., 2005. Human movement analysis using stereophotogrammetry: part 2. instrumental errors. Gait Posture 21, 197-211.

Cimolin, V., Galli, M., 2014. Summary measures for clinical gait analysis: a literature review. Gait Posture 39, 1005-1010.

Cloete, T., Scheffer, C., 2008. Benchmarking of a full-body inertial motion capture system for clinical gait analysis. In: 2008 30th Annual International Conference of the IEEE Engineering in Medicine and Biology Society. IEEE, pp. 4579-4582.

Conley, S., Rosenberg, A., Crowninshield, R., 2007. The female knee: anatomic variations. J. Am. Acad. Orthop. Surg. 15 (Suppl. 1), S31-S36.

Cuesta-Vargas, A.I., Galán-Mercant, A., Williams, J.M., 2010. The use of inertial sensors system for human motion analysis. Phys. Ther. Rev. 15, 462-473.

Davis, R.B., Ounpuu, S., Tyburski, D., Gage, J.R., 1991. A gait analysis data collection and reduction technique. Hum. Mov. Sci. 10, 575-587.

de Bruin, E.D., Hartmann, A., Uebelhart, D., Murer, K., Zijlstra, W., 2008. Wearable systems for monitoring mobility-related activities in older people: a systematic review. Clin. Rehabil. 22, 878-895.

Della Croce, U., Leardini, A., Chiari, L., Cappozzo, A., Della Croce, U., Leardini, A., Chiari, L., 2005. Human movement analysis using stereophotogrammetry. Gait Posture 21, 226-237.

Doi, T., Hirata, S., Ono, R., Tsutsumimoto, K., Misu, S., Ando, H., 2013. The harmonic ratio of trunk acceleration predicts falling among older people: results of a 1year prospective study. J. NeuroEng. Rehabil. 10, 7.

Evans, J.D., 1996. Straightforward Statistics for the Behavioral Sciences. Brooks/Cole Pub. Co, Pacific Grove.

Faber, G.S., Chang, C.-C., Rizun, P., Dennerlein, J.T., 2013. A novel method for assessing the 3-D orientation accuracy of inertial/magnetic sensors. J. Biomech. 46, 2745-2751.

Fernando, M.E, Crowther, R.G. Cunningham, M. Lazzarini, P.A Sangla, K.S. Golledge, J., 2015. Lower limb biomechanical characteristics of patients with neuropathic diabetic foot ulcers: the diabetes foot ulcer study protocol. BMC Endocr. Disord. 15, 59 .

Hara, R., Sangeux, M., Baker, R., McGinley, J., 2014. Quantification of pelvic soft tissue artifact in multiple static positions. Gait Posture 39, 712-717.

Hopkins, W.G., 2000. Measures of reliability in sports medicine and science. Sports Med. 30, 1-15.

Iosa, M., Picerno, P., Paolucci, S., Morone, G., 2016. Wearable inertial sensors for human movement analysis. Expet Rev. Med. Dev. 13, 641-659.

Kavanagh, J.J., Menz, H.B., 2008. Accelerometry: a technique for quantifying movement patterns during walking. Gait Posture 28, 1-15.

Kroll, P., Arnofsky, S., Peckham, S., Rabinowitz, A., 2000. The relationship between lumbar lordosis and pelvic tilt angle. J. Back Musculoskelet. Rehabil. 14, 21-25.

Leardini, A., Chiari, L., Della Croce, U., Cappozzo, A., 2005. Human movement analysis using stereophotogrammetry: part 3. Soft tissue artifact assessment and compensation. Gait Posture 21, 212-225.

Leibbrandt, D., Louw, Q., 2017. Kinematic factors associated with anterior knee pain during common aggravating activities: a systematic review. Phys. Ther. Rev. 22 34-47.

Levine, D., Whittle, M.W., 1996. The effects of pelvic movement on lumbar lordosis in the standing position. J. Orthop. Sports Phys. Ther. 24, 130-135. https:// doi.org/10.2519/jospt.1996.24.3.130.

McGinley, J.L., Baker, R., Wolfe, R., Morris, M.E., 2009. The reliability of threedimensional kinematic gait measurements: a systematic review. Gait Posture 29, 360-369.

Meldrum, D., Shouldice, C., Conroy, R., Jones, K., Forward, M., 2014. Test-retest reliability of three dimensional gait analysis: including a novel approach to visualising agreement of gait cycle waveforms with Bland and Altman plots. Gait Posture 39, 265-271.

Monaghan, K., Delahunt, E., Caulfield, B., 2007. Increasing the number of gait trial recordings maximises intra-rater reliability of the CODA motion analysis system. Gait Posture 25, 303-315. https://doi.org/10.1016/J.GAITPOST.2006.04.011.

Nüesch, C., Roos, E., Pagenstert, G., Mündermann, A., 2017. Measuring joint kinematics of treadmill walking and running: comparison between an inertial sensor based system and a camera-based system. J. Biomech. 57, 32-38.

Peters, A., Galna, B., Sangeux, M., Morris, M., Baker, R., 2010. Quantification of soft tissue artifact in lower limb human motion analysis: a systematic review. Gait Posture 31, 1-8.

Picerno, P., 2017. 25 years of lower limb joint kinematics by using inertial and magnetic sensors: a review of methodological approaches. Gait Posture 51, 239-246.

Picerno, P., Cereatti, A., Cappozzo, A., 2008. Joint kinematics estimate using wearable inertial and magnetic sensing modules. Gait Posture 28, 588-595.

Preece, S.J., Willan, P., Nester, C.J., Graham-Smith, P., Herrington, L., Bowker, P., 2008. Variation in pelvic morphology may prevent the identification of anterior pelvic tilt. J. Man. Manip. Ther. 16, 113-117.

Robert-Lachaine, X., Mecheri, H., Larue, C., Plamondon, A., 2017. Accuracy and repeatability of single-pose calibration of inertial measurement units for whole-body motion analysis. Gait Posture 54, 80-86.

Roiz, R. de M., Cacho, E.W.A., Pazinatto, M.M., Reis, J.G., Cliquet Jr., A., BarasneviciusQuagliato, E.M.A., 2010. Gait analysis comparing Parkinson's disease with 
healthy elderly subjects. Arq Neuropsiquiatr 68, 81-86.

Schache, A.G., Baker, R., Lamoreux, L.W., 2006. Defining the knee joint flexion-extension axis for purposes of quantitative gait analysis: an evaluation of methods. Gait Posture 24, 100-109.

Seidel, D.H., D'Souza, S.F., Alt, W.W., Wachowsky, M., 2015. Comparison of an inertial sensor based motion measurement system with a 3D-reflex marker based motion capture system. Gait Posture 42, S75.

Shull, P.B., Jirattigalachote, W., Hunt, M.A., Cutkosky, M.R., Delp, S.L., 2014. Quantified self and human movement: a review on the clinical impact of wearable sensing and feedback for gait analysis and intervention. Gait Posture 40, 11-19.

Stratford, P.W., Goldsmith, C.H., 1997. Use of the standard error as a reliability index of interest: an applied example using elbow flexor strength data. Phys. Ther. 77, $745-750$.

van Schooten, K.S., Pijnappels, M., Rispens, S.M., Elders, P.J.M., Lips, P., van Dieën, J.H., 2015. Ambulatory fall-risk assessment: amount and quality of dailylife gait predict falls in older adults. Journals Gerontol Ser A Biol Sci Med Sci 70,
$608-615$

Weir, J.P., 2005, Quantifying test-retest reliability using the intraclass correlation coefficient and the SEM. J. Strength Condit Res. 19, 231.

Wilken, J.M., Rodriguez, K.M., Brawner, M., Darter, B.J., 2012. Reliability and minimal detectible change values for gait kinematics and kinetics in healthy adults. Gait Posture 35, 301-307.

Wu, G., Siegler, S., Allard, P., Kirtley, C., Leardini, A., Rosenbaum, D., Whittle, M. D'Lima, D.D., Cristofolini, L., Witte, H., Schmid, O., Stokes, I., Standardization and Terminology Committee of the International Society of Biomechanics, 2002. ISB recommendation on definitions of joint coordinate system of various joints for the reporting of human joint motion-part I: ankle, hip, and spine. Int. Soc. BioMech.J Biomech 35, 543-548. https://doi.org/10.1016/s0021-9290(01) 00222-6.

Yu, B., Gabriel, D., Noble, L., An, K.-N., 1999. Estimate of the optimum cutoff frequency for the Butterworth low-pass digital filter. J. Appl. Biomech. 15, 318-329. 